\title{
Diseases associated with ocular surface abnormalities: the importance of reflex tearing
}

\author{
Kazuo Tsubota, Minako Kaido, Yukiko Yagi, Tsutomu Fujihara, Shigeto Shimmura
}

\begin{abstract}
Aim-To investigate the correlation between tear function tests and ocular surface integrity in patients with dry eye. Methods-297 dry eye patients (55 Sjögren's syndrome, two male and 53 female, average age 52.4 (SD 15.0) years, and 242 non-Sjögren's syndrome, 41 male and 201 female, average age 53.5 (14.1) years) were examined. The following tear function tests were performed: (1) cotton thread test, (2) Schirmer test with topical anaesthesia, (3) Schirmer test without anaesthesia, (4) Schirmer test with nasal stimulation, (5) tear clearance test, and (6) tear break up time (BUT). The ocular surface was evaluated by rose bengal and fluorescein staining. Correlation analysis was performed between each tear function index and vital staining scores.

Results-Among the six tear function tests, the Schirmer test with nasal stimulation correlated most with both of the vital stains $(\rho=0.530$ for rose bengal and 0.393 for fluorescein). The Schirmer test with or without anaesthesia correlated slightly with rose bengal staining, whereas tear clearance test and tear break up time slightly correlated with fluorescein staining.

Conclusion-Vital staining of the ocular surface correlates most with reflex tearing measured by the Schirmer test with nasal stimulation.
\end{abstract}

(Br f Ophthalmol 1999;83:89-91)

Department of Ophthalmology, Tokyo Dental College, Chiba, Japan

K Tsubota

M Kaido

Y Yagi

T Fujihara

S Shimmura

Department of Ophthalmology, Keio University School of Medicine, Tokyo, Japan

K Tsubota

S Shimmura

Correspondence to: Kazuo Tsubota, MD, Department of Ophthalmology, Tokyo Dental College, 11-13 Sugano 5 Chome,

Ichikawa-shi, Chiba, Japan 272-8513.

Accepted for publication 5 August 1998
A wide variety of disorders are associated with ocular surface abnormalities including dry eye, simple mechanical abrasions such as contact lens wear, infections, chemical and thermal burns, and autoimmune diseases such as ocular pemphigoid and Stevens-Johnson syndrome. Alterations of the ocular surface usually start with minor changes such as shortening of tear break up time (BUT), which subsequently progresses to squamous metaplasia accompanied by rose bengal and fluorescein staining. The end stage involves corneal neovascularisation and persistent epithelial defects as a result of stem cell dysfunction. ${ }^{1-3}$

Among the various diseases affecting the ocular surface, dry eye is the most common condition. ${ }^{4}$ In standard outpatient clinics, it has been reported that $15-30 \%$ of new patients are affected by dry eye..$^{5}$ Although a decrease in tear production is a common condition in many types of dry eye, the severity of ocular surface lesions varies greatly from disease to disease. ${ }^{1}$ A clear correlation has not been found between tear functions tests such as the Schirmer test and tests of ocular surface integrity such as rose bengal and fluorescein staining. ${ }^{6}$

In previous reports, we have emphasised the importance of reflex tearing for the maintenance of the ocular surface. ${ }^{78}$ Although reflex tearing is not compromised in most types of dry eyes, autoimmune related diseases such as Sjögren's syndrome often result in the destruction of the lacrimal gland. Since tear contains factors such as epidermal growth factor (EGF) or vitamin A that are required for the proper proliferation differentiation of the ocular surface epithelium, ${ }^{9-12}$ the lack of tear components may severely compromise ocular surface integrity. We have thus speculated that measurement of reflex tears is the most important factor in the assessment of the ocular surface among the various tear function tests available. ${ }^{13}$

In this paper, the tear function and ocular surface in dry eye patients was evaluated by various techniques. Correlation analysis was performed between each tear function test and vital stain to observe for any consistent patterns.

\section{Patients and methods}

DRY EYE PATIENTS

The diagnosis of dry eye was made using the following three criteria as previously reported $^{14}$ : (1) symptoms of dry eye, (2) abnormalities of tear dynamics determined by the Schirmer test $(<5 \mathrm{~mm}$ after 5 minutes), clearance test $(8 \times)$, cotton thread test $(<10$ $\mathrm{mm}$ after 5 minutes), and tear break up time (BUT, 5 seconds), (3) abnormalities of ocular surface determined by rose bengal $(>3+)$ or fluorescein vital staining $(>3+)$. Vital staining was performed using a fixed concentration and volume of dye in order to obtain consistent results. ${ }^{15} 16$ Patients with symptoms and positive findings in tear function tests or staining scores were diagnosed as dry eye, and the diagnosis of Sjögren's syndrome was made according to Fox's criteria. ${ }^{17}$

A total of 297 dry eye patients ( 55 with Sjögren's syndrome (SS) (two males, 53 females, average age of 52.4 (SD 15.0) years) and 242 non-Sjögren's (non-SS) dry eye patients (41 males, 201 females, average age of 53.5 (14.1) years)) were recruited for this study. Among the 242 non-SS dry eye patients, 103 had low levels $(<160)$ of autoantibody such as rheumatoid arthritis factor (RA) or antinuclear antibody (ANA), but did not match the diagnosis criteria of Sjögren's syndrome, and were therefore classified as autoimmune positive dry eye. 
Table 1 Correlation between vital staining and tear function tests (as a whole)

\begin{tabular}{|c|c|c|}
\hline & Rose bengal score & Fluorescein score \\
\hline & Right eye & Right eye \\
\hline Cotton thread test & $\begin{array}{l}\rho=-0.087 \\
(p=0.1372)\end{array}$ & $\begin{array}{l}\rho=0.021 \\
(p=0.7224)\end{array}$ \\
\hline Schirmer test with anaesthesia & $\begin{array}{l}\rho=-0.197 \\
(p=0.0007)\end{array}$ & $\begin{array}{l}\rho=-0.043 \\
(p=0.4645)\end{array}$ \\
\hline Schirmer test without anaesthesia & $\begin{array}{l}\rho=-0.344 \\
(p<0.0001)\end{array}$ & $\begin{array}{l}\rho=-0.197 \\
(p=0.0020)\end{array}$ \\
\hline Schirmer test with nasal stimulation & $\begin{array}{l}\rho=-0.530 \\
(p<0.0001)\end{array}$ & $\begin{array}{l}\rho=-0.393 \\
(p<0.0001)\end{array}$ \\
\hline Tear clearance test & $\begin{array}{l}\rho=-0.161 \\
(p=0.0059)\end{array}$ & $\begin{array}{l}\rho=-0.283 \\
(p<0.0001)\end{array}$ \\
\hline Tear break up time & $\begin{array}{l}\rho=-0.174 \\
(p=0.0043)\end{array}$ & $\begin{array}{l}\rho=-0.332 \\
(p<0.0001)\end{array}$ \\
\hline
\end{tabular}

Each value shows the Spearman rank correlation $(\rho)$ and probability $(p)$.

Eighty five patients had simple dry eye with positive ocular surface staining, but without evidence of autoantibodies. ${ }^{1}$ The remaining 54 patients had only decreased tear break up time (BUT) associated with symptoms of dry eye without positive vital staining. ${ }^{14}$

TEAR EVALUATION

The Schirmer value with anaesthesia and the tear clearance rate (TCR) were measured 5 minutes after instilling a $10 \mu \mathrm{l}$ drop of $0.5 \%$ fluorescein and $0.4 \%$ oxybuprocaine hydrochloride solution into the conjunctival sac. ${ }^{18}$ The Schirmer value was measured, and TCR was determined by the staining intensity of the filter which was compared with a dilution standard graded from $1 \times$ to $256 \times$. The Schirmer test without topical anaesthesia was also performed, while maximum tear production was evaluated by the Schirmer test with nasal stimulation, ${ }^{8}$ which measures the maximal tear secretion from the ipsilateral eye. Briefly, the patients were examined by the simple Schirmer test for 5 minutes without topical anaesthesia. Then a cotton swab was inserted into the patient's nasal cavity, ${ }^{8}$ and the Schirmer test was repeated for 5 minutes using a $75 \mathrm{~mm}$ long Schirmer paper strip while the cotton swab was kept in place. An increase in tear production is observed when the lacrimal gland is intact. The Schirmer test with nasal stimulation is performed after the confirmation of low Schirmer results without nasal stimulation. This test is usually performed several minutes after the first testing on the same day or on the next visit if the patient did not have the time to have the second test. All tear evaluation tests were performed by the same examiner (YG).

\section{OCULAR SURFACE EVALUATION}

The ocular surface was examined by the double staining method. Two $\mu \mathrm{l}$ of preservative-free solution consisting of $1 \%$ rose bengal and $1 \%$ fluorescein dye were applied to the conjunctival sac by micropipette. ${ }^{15} 16$ The severity of rose bengal staining was recorded in the temporal and nasal conjunctiva and cornea, and then quantified on a scale of 0 to 3 points. Thus, the maximum score obtained from the staining of one eye is 9 . Fluorescein staining was also rated from 0 to 9 , but only in the cornea. All of the evaluation was done by one examiner (KT).
DATA ANALYSIS

Spearman's rank correlation test was performed between each vital staining score (rose bengal and fluorescein) and tear function tests.

\section{Results}

Table 1 shows the results of correlation analysis between vital staining scores and tear function variables. Analysis of rose bengal scores revealed that the correlation coefficient $(\rho)$ was greater than -0.5 only between the rose bengal score and the Schirmer test with nasal stimulation. Although other tear function tests also demonstrated some correlation with rose bengal scores, they were relatively small. The Schirmer test with and without anaesthesia had correlation coefficients of -0.197 and -0.344 respectively, whereas the cotton thread test, tear clearance test, and tear break up time had $\rho$ values of less than -0.2 .

Correlation between fluorescein staining and tear function tests demonstrated a similar tendency. Maximal correlation was observed in the Schirmer test with nasal stimulation $(\rho=$ -0.393) followed by tear break up time ( $\rho=$ $-0.332)$ and tear clearance $(\rho=-0.283)$. The cotton thread test, the Schirmer test with and without anaesthesia had values of less than -0.2 .

Of all the tear function variables, the Schirmer test with nasal stimulation correlated most with both rose bengal and fluorescein staining, while the Schirmer test with and without anaesthesia slightly correlated with rose bengal staining. Tear clearance and BUT were associated more with fluorescein staining than rose bengal staining. The cotton thread test did not show any correlation with either dye.

\section{Discussion}

This study demonstrated the importance of reflex tearing in the primary assessment of patients with dry eye. Among all the various tear function tests examined, the results of the Schirmer test with nasal stimulation was the only test that showed acceptable correlation with vital staining.

We have previously reported that reflex tearing is dramatically decreased in SS dry eye and that the ocular surface tends to be more compromised than in non-SS dry eye. ${ }^{18}$ Although the Schirmer test without anaesthesia has been considered to reflect reflex tearing, many patients with low values in this test are capable of producing substantial amounts of reflex tears by other stimuli such as nasal stimulation. This phenomenon suggests that the Schirmer test without anaesthesia does not accurately measure maximal tear secretion. In contrast, the Schirmer test with nasal stimulation shows greater values in patients with residual lacrimal function, and correlates well with lacrimal gland destruction observed in pathological sections. ${ }^{18}$ Since the Schirmer test with nasal stimulation reflects the actual function of the lacrimal gland whose purpose is to protect the ocular surface, it is not surprising that the results of the Schirmer test with nasal 
stimulation correlated most with rose bengal and fluorescein staining of the ocular surface.

Since the Schirmer test is a diagnostic criterion for dry eye, and rose bengal staining is a prominent finding in such eyes, it has been presumed that a correlation exists between tear function measured by Schirmer test and ocular surface abnormalities detected by the vital dyes. However, there is still a controversy as to the correlation between the Schirmer test and ocular surface conditions. Paschides et al reported that there was no correlation between the Schirmer test results and squamous metaplasia of the ocular surface. ${ }^{19}$ To our knowledge, there are no definite reports providing evidence of any correlation between tear function tests and ocular surface abnormalities. This may be due to the relatively small number of patients examined, or the variation in test results which is always a factor in such studies. The present study attempted to eliminate these variables by using micropipettes to instil dyes, recruiting a large number of patients, as well as limiting the examiner and investigator to a single person to assure maximal objectivity. Although the correlation coefficients observed in our study are relatively small, it is possible to say that under the clinical conditions given, a slight correlation exists between the Schirmer test and vital dye staining.

It is interesting to note that all types of the Schirmer test correlated more with rose bengal staining than fluorescein staining, whereas tear clearance and tear BUT correlated more with fluorescein staining than rose bengal. Although the cotton thread test is believed to reflect the amount of accumulated tears in the cul de sac at the time of measurement, no correlation was observed in this study. Since rose bengal staining reflects epithelium cells deprived of mucin, whereas fluorescein staining reflects the destruction of cell to cell junctions, the two factors reflect different pathological conditions..$^{20}$ Conjunctival mucin expression may be related more to the lack of tear components, while corneal permeability may be related more to the accumulation of inflammatory cytokines or cytotoxic factors as a result of poor clearance.
In conclusion, we have demonstrated that the Schirmer test with nasal stimulation best reflects ocular surface abnormalities among the various tear functional variables available. Although this test is not common in standard clinical practice, it is recommended for better understanding of dry eyes and lacrimal gland function owing to the simplicity and reproducibility of the procedure. The Schirmer test with nasal stimulation is the most reliable tear function variable that can be used to screen potential SS patients before costly serological tests.

1 Tsubota K, Toda I, Yagi Y, et al. Three different types of dry eye syndrome. Cornea 1994;13:202-9.

2 Tseng S. Staging of conjunctival squamous metaplasia by impression cytology. Ophthalmology 1985;92:728-33.

3 Tseng S. Concept and application of limbal stem cells. Eye 1989;3:141-57.

4 Lemp MA. Report of the national eye institute/industry workshop on clinical trials in dry eyes. CLAO $71995 ; 21$ : 221-32.

5 Hikichi T, Yoshida A, Fukui Y, et al. Prevalence of dry eye in Japanese eye centers. Graefes Arch Clin Exp Ophthalmol 1995;233:555-8.

6 Lemp MA. Recent developments in dry eye management. Ophthalmology 1987;94:1299-305.

7 Tsubota K. The importance of the Schirmer test with nasal stimulation [letter]. Am f Ophthalmol 1991;111:106-8.

8 Tsubota K, Xu K, Fujihara T, et al. Decreased reflex tearing is associated with lymphocytic infiltration in lacrimal glands. F Rheumatol 1996;23:313-20.

9 Ohashi Y, Motokura M, Kinoshita Y, et al. Presence of epidermal growth factor in human tears. Invest Ophthalmol Vis Sci 1989;30:1879-87.

10 Ubels J, Loley K, Rismondo V. Retinol secretion by the lacrimal gland. Invest Ophthalmol Vis Sci 1986;27:1261-9.

11 Wilson S. Lacrimal gland epidermal growth factor production and the ocular surface. Am f Ophthalmol 1991;111: tion and 5 .

12 Van Setten G, Viinikka L, Tervo T. Epidermal growth factor is a constant component of normal human tear fluid. Graefes Arch Clin Exp Ophthalmol 1989;227:184-7.

13 Tsubota K. New approaches in dry eye management: supplying missing tear components to the ocular surface epithelium. In: Kinoshita S, Ohashi Y, eds. Current Opinions in the Kyoto Cornea Club. Amsterdam: Kugler Publications, 1997;1:27-32.

14 Toda I, Shimazaki J, Tsubota K. Dry eye with only decreased tear break-up time is sometimes associated with allergic conjunctivitis. Ophthalmology 1995;102:302-9.

15 Toda I, Tsubota K. Practical double vital staining for ocular surface evaluation. Cornea 1993;12:366-7.

16 Van Bijsterveld OP. Diagnostic tests in the sicca syndrome. Arch Ophthalmol 1969;82:10-14.

17 Fox R, Robinson C, Curd J, et al. Sjogren's syndrome: proposed criteria for classification. Arthritis Rheum 1986;29: 577-83.

$18 \mathrm{Xu} \mathrm{K}$, Yagi Y, Toda I, et al. Tear function index: a new measure of dry eye. Arch Ophthalmol 1995;113:84-8.

19 Paschides CA, Petroutsos G, Psilas K. Correlation of conjunctival impression cytology results with lacrimal function and age. Acta Ophthalmol (Copenh) 1991;69:4225 .

20 Feenstra R, Tseng S. Comparison of fluorescein and rose bengal staining. Ophthalmology 1992;99:605-17.

21 Feenstra R, Tseng S. What is actually stained by rose bengal? Arch Ophthalmol 1992;110:984-93. 